\title{
A Method of Cognitive Training of Medical Students and Residents
}

\author{
Bobby Desai1 ${ }^{1}$ Alpa Desai ${ }^{2}$ \\ ${ }^{1}$ University of Central Florida, Orlando, FL, USA \\ ${ }^{2}$ University of Florida, Gainesville, FL, USA \\ Email: ocalaemergencymedicine@gmail.com
}

How to cite this paper: Desai, B., \& Desai, A. (2018). A Method of Cognitive Training of Medical Students and Residents. Creative Education, 9, 1377-1388.

https://doi.org/10.4236/ce.2018.99102

Received: May 29, 2018

Accepted: July 21, 2018

Published: July 24, 2018

Copyright (c) 2018 by authors and Scientific Research Publishing Inc. This work is licensed under the Creative Commons Attribution International License (CC BY 4.0).

http://creativecommons.org/licenses/by/4.0/

\begin{abstract}
Beginning learners in medicine face a daunting challenge of utilizing information gained in the first two years of medical school in a clinical setting in the latter half of their medical school and into residency. Constant interruptions and lack of knowledge are but two of the factors that may hamper the early clinician's ability to safely and efficiently care for patients. Thus, a system that organizes thought into specific compartments-compartmentalization may benefit the learner by separating tasks into smaller, more specific objectives to complete prior to moving on in the diagnostic algorithm. Developing a system that utilizes compartmentalization may ease the transition into clinical practice using simple and effective tools that allow the beginning physician to mitigate the invariable distractions and interruptions that occur daily to allow for the safe practice of medicine.
\end{abstract}

\section{Keywords}

Cognition, Interruptions, Multitasking, Workload, Cognitive Load Theory, Compartmentalization, Residents, Medical Students

\section{Introduction}

Emergency medicine is the medical specialty dedicated to the diagnosis and treatment of unforeseen illness or injury. It encompasses a unique body of knowledge as set forth in the "Model of the Clinical Practice of Emergency Medicine", and its practice includes the initial evaluation, diagnosis, treatment, coordination of care among multiple providers, and disposition of any patient requiring expeditious medical, surgical, or psychiatric care (Counselman, Babu, Edens, Gorgas, Hobgood, Marco et al., 2017). As such, it is a specialty that has adopted the adage of "anyone, anything, anytime". For the student or new 
learner, this field (as well as any field in medicine) initially can be overwhelming. The field poses a significant cognitive challenge for the new learner and may be fraught with error due to multiple factors. In fact, in 2001, researchers have laid out specific characteristics of the Emergency Department (ED) that predisposed to medical error (Croskerry \& Sinclair, 2001). Along with the required high cognitive load, other reasons cited for medical error included inexperience, poor or inadequate decision-making ability, and constant interruptions. The need to multitask and the inherent workload demands in emergency medicine (as well as other medical specialties) also play a role in patient care outcomes. Thus, there is a need to train all learners in specific methodologies to limit errors, provide timely and efficient medical care and foster learning.

\section{The Multitasking Quandary and the Role of Interruptions}

Since there is, by definition, no set schedule for either patient arrivals or acuity and since time constraints (including wait times, length of stay metrics, etc.) are becoming the new norm in the practice of medicine, the new learner in medicine must in rapid order become skilled at: assessment of a new patient, potentially determine placement of that patient (urgent care, etc.), or even the waiting room). They must also begin the process of workup, perform diagnostic and therapeutic procedures, call consultants if required, and ultimately disposition the patient appropriately. Therefore, multitasking in medicine has become a core skill. The term "multitasking" refers to the management of multiple simultaneous tasks. This skill is applicable to most specialties in medicine and is not solely limited to any one specialty. For example, during the course of day, a busy Family Medicine provider must, juggle a fully booked patient schedule, address patient phone calls and medication refills, field questions from nurses, all while teaching medical students and residents if working in an academic environment. In fact, there have been studies showing issues with patient safety due to multitasking (Archana, Forogh, Osman, Robert, Zhang, \& Vimla, 2007). Thus, in order to function effectively in most practice environments, must learn the ability to multitask effectively and safely. Ultimately, the trainee must learn how to both perform two or more distinct tasks rapidly and effectively, enhancing the completion of the tasks while decreasing any additional cognitive load.

There are multiple interruptions that occur in the daily practice of medicine. From verbal interruptions, phone calls, to computer use, to electrocardiograms that require signature, interruptions are a daily part of the work life of the physician and each interruption raises the potential for errors occurring (Rhodes, McFarland, Finch, \& Johnson, 2001; Chisholm, Collison, \& Nelson, 2000). Constant distractions may cause loss of focus, affect critical thinking to the point where analysis of data may become faulty (Chisholm, Collison, \& Nelson, 2000). All may potentially lead to medical error and patient safety issues. In addition to physicians, ancillary staff in a medical practice also has issues with interruptions. Interruptions, as well as work overload were cited by nursing staff as significant 
stressors (Brown, 1995). The role of interruptions is critical in medicine due to the fact that they distract the provider from the current task and lead to difficulty in concentration when returning to that task. Some researchers have labeled these interruptions as a "substantial threat to thought flow and job efficiency" (Hymel \& Severyn, 1999).

In general, interruptions can be classified as "external" versus "internal". External interruptions include those already mentioned. Internal interruptions are those that occur when the physician's mind wanders to a newer task rather than being interrupted by an external factor. Whether internal or external, all of these influence completion (or lack thereof) of the providers activities and may potentially decrease the accuracy of each individual task, thus potentially increasing error (Skaugset, Farrell, Carney, Wolff, Santen, Perry, \& Cico, 2016).

We can use the intrinsic ability of the brain to separate or divide a large task into smaller, more easily completed components, creating a method of training learners to focus on these smaller tasks of a greater whole. If one can focus on these smaller tasks, this may decrease incompletion of a group of tasks due to interruptions.

\section{The Role of Workload}

Cognitive training is important in the context of physician workload for several reasons. The two most relevant include medical error and graded responsibility. The amount of work placed on a physician has been directly attributed as a significant cause of preventable medical error. This has led to the implementation of resident duty hours. Excessive workload is thought to increase physician fatigue which may result in preventable medical errors (Jagsi, Weinstein, Shapiro, Kitch, Dorer, \& Weissman, 2008; Kohn, Corrigan, \& Donaldson, 2000). Increasing workload is thought to hinder patient care in multiple ways. The first is due to the inability to perform an adequate history and physical exam due to time constraints. In a busy clinical setting such as an ED, the role of interruptions and the need to be cognizant of the intrinsic surroundings inherent there plays a significant role in the lack of a thorough history and physical exam. For example, during a routine interview, there may be a concurrent trauma or medical code, necessitating the immediate attention of the physician. Another consideration of workload demands is that in those circumstances where there is "time" (for example, when alerted that a trauma code is 10 minutes away-enough time to perform a cursory history and physical exam, but not enough to perhaps gather the nuances), the physician may potentially order unnecessary items including diagnostic testing and inappropriate consultation. This may, in theory, lead to a cascade of significant unintended consequences where a test result requires the performance of yet another test to "prove" a positive or negative depending on the circumstances. Workload demands also may hinder the safe performance of complicated procedures (which may themselves lead to potential medical error). Lastly, and perhaps most important, workload 
demands may preclude the ability to fully discuss treatment options and share decision-making with the patient and their family (Michtalik, Yeh, Pronovost, \& Brotman, 2013). In short, the higher the workload, the higher the potential for error.

Another reason why cognitive training is important is that in spite of the potential for medical error, increasing workload and graded autonomy is important for the successful maturation of a learner to a seasoned attending physician. The concept of graded autonomy is important for the learner in medicine in order to master efficiently manage a busy medical practice. When becoming independent, one learns to function by making decisions and performing procedures without significant supervision. However, in order to do this the learner must develop a cognitive schema in order to proficiently manage multiple patients safely and effectively. Without the challenging transition from resident to independent practice, "transition shock" may present ultimately causing decreased productivity, efficiency and lack of perceived achievement (Duchscher, 2009; Tweed, Bagg, Child, Wilkinson, \& Weller, 2010). Lastly, the development of this schema to deal with increasing workload is important because as the physician's load increases, the overall unpredictable environment becomes more stressful which is a risk factor for both burnout and a decreased quality of work life (Kirmeyer, 1988; Bragard, Dupuis, \& Fleet, 2015).

\section{Cognition Basics}

Emergency Medicine (and Medicine in general), requires several skills to be mastered to provide safe and effective care. Per Croskerry (Croskerry, 2000), this skillset includes cognitive, procedural, and affective skills. These competencies are generally utilized in concert in order to effectively provide safe patient care and to reduce medical errors. In addition to these general categories, other processes or skills that have been discussed in the literature include social interactions with others (critical in most areas of medicine), metacognition (thinking about thinking), the specific location or setting of learning (e.g. operating room, ED, or primary care clinic), and cognitive load theory (Young, Van Merrienboer, Durning, \& Ten Cate, 2014).

Theories of learning are numerous, come from multiple academic specialties, yet there is no one specific theory that is applicable to all field, and thus educators must choose from those most applicable to them.

In terms of medical education, cognition is involved in high order medical decision-making. In fact, it can be argued that specific cognitive abilities are required that play a role in the performance of specific human behaviors and skills. An example lies in a three-level model of cognition. In this model, cognition has three levels of complexity. These include skill-based (procedures), rule-based (decision rules, e.g. Ottawa ankle rules), and knowledge-based (decision-making and diagnostic reasoning). The knowledge-based skill is highest order skill achievable and includes interpretation and extrapolation of information in a 
specific context (Rasmussen \& Jensen, 1974). Experience gained by modalities such as simulation and "real world" work allows the knowledge-based skillset one to revert to a less cognitively intense skill set. In this case, experience equals knowledge. Lastly, lack of development of these skills may lead to medical error and lack of expertise; however, without time and experience, these skills may not be fully and robustly developed.

\section{Cognitive Load Theory, Memory, and the Development of Expertise}

Cognitive load theory was first described in 1988 and specifically deals with memory. It describes the integration of memory systems (specifically working and long-term memory-the other subsystem of this component is sensory), the types of cognitive load placed on working memory, and the overall process of learning. A summary of this theory follows: data enters via the sensory memory system which can analyze a large amount of auditory and visual information, but only for a short time. This information is subsequently sent to working memory. Unfortunately, working memory, as discussed by Miller in 1956, does not have a large capacity; working memory cannot process more than seven distinct units at one time and for only about 15 - 30 seconds (Miller, 1956). Finally, per cognitive load theory, this information is restructured and stored in long term memory where it is recovered when needed. In terms of general cognition and the three-level model, utilization of long term memory can be likened to a less cognitively intense skill.

As previously mentioned, the development of any expertise will occur over time. Expertise requires both the acquisition of knowledge as well as the experience in applying that gained knowledge to specific tasks (17). Per Chi, experts will generate better solutions to problems, they will elucidate clues that others do not, will analyze problems in a more qualitive way, potentially choose better strategies for problem-solving, and finally will use any available information in a more efficient cognitive way (Chi, 2006) Experts will have a more robust long-term memory when in their professional domain and will utilize their developed schemata more efficiently in order to arrive at solutions more easily (Ericsson, 2006).

In terms of clinical reasoning, an expert utilizes two modalities. The first is a rapid production of thought that will typically emerge as a known pattern, and the second is a slower, more analytic, process (Eva, 2005). For those problems that are routine, the recognition of a pattern is the dominant modality and typically requires less cognitive effort. For those problems that are new and not quickly recognized, the role of analytic reasoning becomes dominant.

Beginners typically cannot recognize patterns (due to lack of knowledge) and cannot draw conclusions based upon these patterns and therefore must utilize analytic reasoning. This is where the beginning physician may benefit from specific cognitive training in order to develop known patterns. 


\section{A Method of Cognitive Training}

We have discussed how the new learner to medicine is faced with enormous challenges when beginning training. The need to develop the skill of multitasking, learn to minimize interruptions, develop one's cognitive ability all while increasing their knowledge base to ultimately make their job easier is paramount to the safe, effective and efficient practice of medicine. Thus, it seems logical to utilize what is already known and develop it further. This is the "initial gestalt" when seeing a patient- "sick or not sick".

In terms of gaining knowledge and applying it clinically, cognitive load theory has been demonstrated to be effective. This theory is eminently applicable to learning in multiple phases of the evolution of a physician, from the so called "book phase" to even clinically, but it is missing some components that are present in the day to day practice of medicine.

The general schema of the cognitive load theory is shown below:

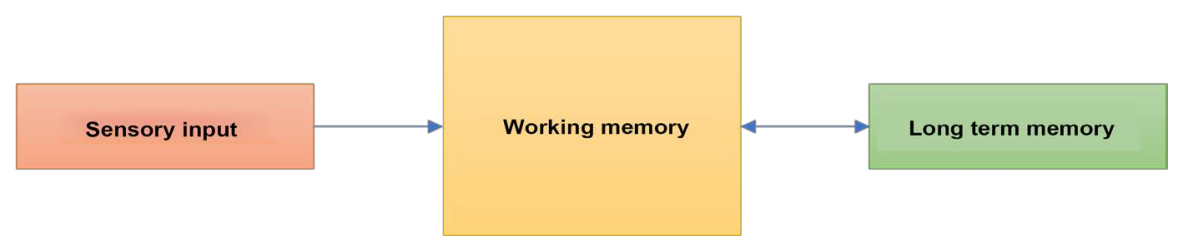

We can modify this basic schema into one more relevant to clinical practice, along with pitfalls.

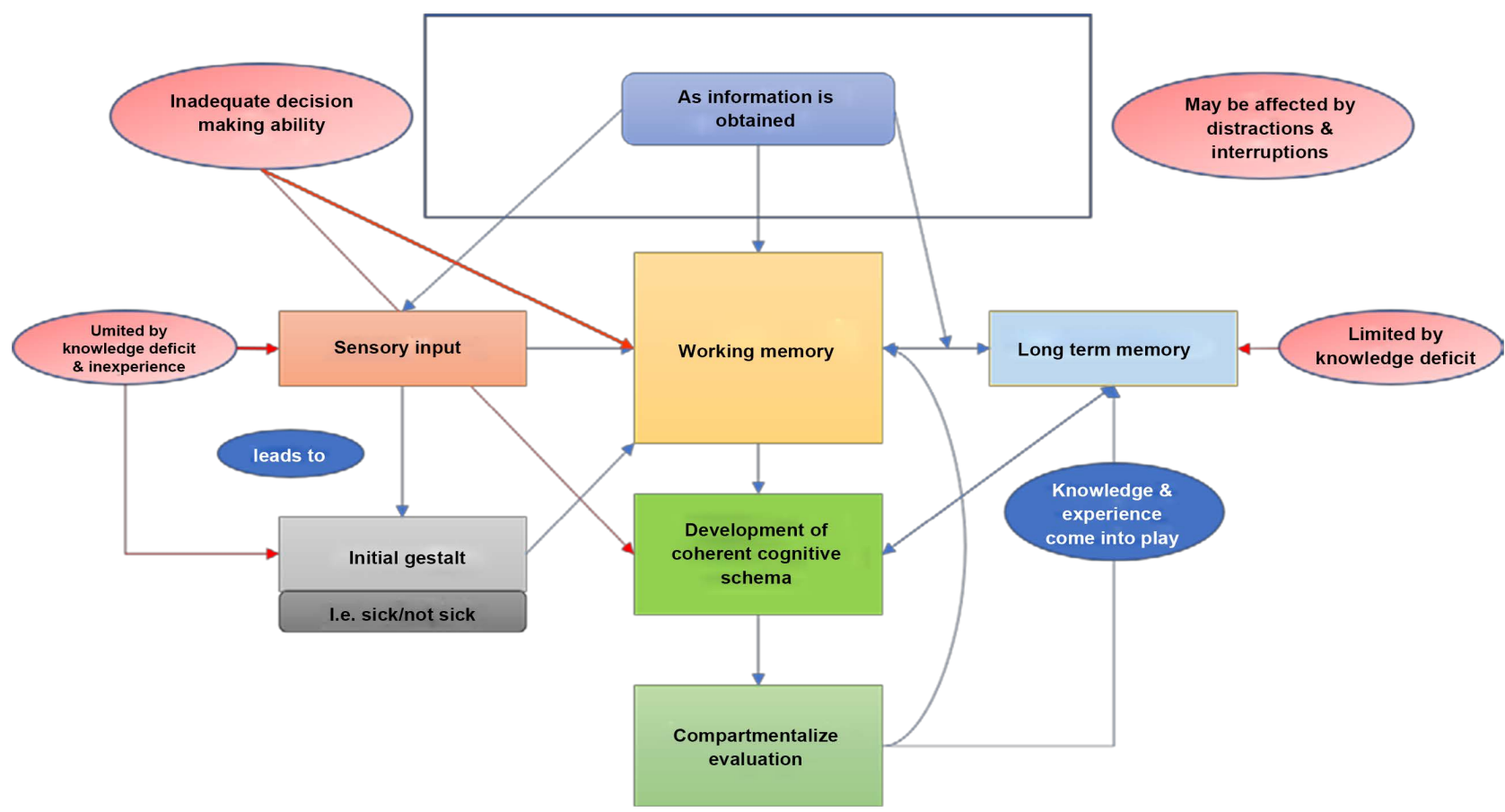

While in theory, this schema may seem ideal to the student learning medicine, it assumes that the learner has the innate capacity to eschew extraneous and ir- 
relevant information; however, this assumes the learner already the long-term knowledge required to do so. Thus, this schema requires some modification to account for the processes inherent to medicine discussed earlier: the roles of multitasking, task interruptions and workload must be considered. In addition, once the development of the cognitive schema is made, and knowing that the contents within the working memory are fleeting, how can the learner use the information gathered to develop a differential diagnosis and plan of action which potentially includes diagnostic tests, imaging, treatment, and ultimately, disposition?

\section{Compartmentalization}

Compartmentalization, or simply stated, the division of thought into specific sections or categories can be utilized in training the beginning learner in medicine. In this method, the beginning learner can utilize the initial sensory input provided by a patient encounter in order to formulate development of the initial gestalt and a coherent cognitive schema that will advance both knowledge and experience. This is essentially the sorting out and prioritization of relatively conflicting demands, placing it within working memory, each in its own "cubby", staying in place until one can call on it. If we can compartmentalize specific aspects of patient care, isolating it from any other challenge learner is dealing with at that time, the learner may apply extreme focus on each compartment which is only necessary for short period of time, which is essentially the limit of the working memory timeframe. The learner may then consider available options in that compartment and subsequently move forward in incremental steps, essentially closing the compartment once progress has been achieved. The learner may then close that compartment and move on to the next one (Blair, 2012). In this manner, as more information is obtained via sensory input and subsequently transferred into working memory, the learner may begin to develop coherent cognitive schema leading to more efficient pathways leading to long-term memory, thus gaining knowledge and experience.

\section{Steps of Compartmentalization}

1) Create compartments for specific tasks that require completion. These may be different for each specialty but each compartment should be specific enough to require completion. Separating one task from another allows one to take control and make conscious decisions on one important item at a time. If an interruption occurs, that compartment may be left open for more thought, or closed due to task completion, but the learner "knows" that they are focusing only on that specific compartment.

2) These streams of thought should be specific for specific tasks. For example, separating laboratory testing from imaging allows the physician to focus solely on that task.

3) Each area should have extreme focus placed on it which may prevent errors 
of omission. In each compartment, the transference from long term memory to working memory will take place, facilitating knowledge retrieval. In addition, there will be a transfer of information from working memory to long term memory which will add both experience and knowledge. Finally, this back and forth between working memory and long-term memory will allow the new learner to develop their own schemas or pathways for future patients with similar issues. In case of the novel presentation, this extreme focus placed upon a single compartment will allow the learner to utilize parts of formerly developed schemata in order to assist in the determination of potential next steps.

4) Transitioning between compartments should occur only if the prior compartment has been thoroughly vetted.

5) The learner can always return to a compartment if a subsequent compartment brings up a thought that was not readily obvious.

6) Finally, utilizing all developed compartments can help the learner "bring it all together", thereby formulating a diagnostic and therapeutic regimen for the patient.

\section{An Example of the General Method of Compartmentalization}

This example is specific for high acuity settings, and it may be used successfully by the learner to begin to establish a diagnostic and therapeutic schema for patient care. It must be noted that each compartment relies on the compartment immediately subsequent.

First, the learner may utilize information provided by emergency medical services (EMS), nursing staff, family members present at bedside, or old medical records. For example, EMS may provide the learner with information on the home condition of the patient they have brought to the ED. As an example, for overdose situations, they may bring the medication bottles with them. They also may obtain history from family members who may have called them. These are important pieces of information that will allow the provider to begin to develop a cohesive plan of action. Nursing staff will provide the medical provider with vital signs, electrocardiograms, blood glucose readings, as well as their own general gestalt of the patient.

The following compartment is that of the appropriate history and physical examination. In an ED setting, this can be entirely a focused one where as in a general practice, this may be more thorough. Without this compartment being thoroughly vetted, further compartments cannot be generated. This compartment is by far the most critical for the new learner. This compartment will dictate thought for all of the ones that follow.

Once the history and physical is obtained, the next compartment one may choose to consider is the differential diagnosis. For example, if the patient presents with complaints of chest pain, what is the differential diagnosis of this patient's pain? Is it cardiac, pulmonary, musculoskeletal, etc.? For novel presentations, this will require retrieval of stored long-term memory given a specific 
sensory input. Working memory will be in play here as well, receiving information in the form of all of the sensory input obtained via the history and physical examination in order to transfer information into long term memory. This compartment is probably the second most important for initial diagnosis and treatment. Without this compartment being thoroughly considered, the learner will find it very difficult to proceed.

Given a litany of multiple differential diagnoses, the next compartment that can be generated by the learner is one dealing with laboratory investigation. As mentioned, separating this from specific imaging allows the learner to focus solely that aspect of care. Given a specific set of differential diagnoses, the learner will begin to have an idea of what laboratory studies may be of benefit in the assessment of the patient. In our example of the patient who presents with chest pain, if the learner believes that this is cardiac in nature, an evaluation of cardiac enzymes most likely should be done. However, if the learner believes that this is musculoskeletal in origin, assessment of cardiac enzymes may not be warranted.

The next compartment may be one considering what imaging may be required for this patient. In our case of the patient with chest pain, is a simple chest $\mathrm{x}$-ray required versus the need for more sophisticated studies such as a computed tomography? Again, this will entirely depend upon the differential diagnoses developed in the second compartment. To reiterate why a complete vetting of the preceding compartments is critical is due to the fact that without an appropriate differential diagnosis stemming from a appropriate history and physical, the learner may be steered into obtaining an imaging modality that is completely inappropriate for the patient's presentation. In our example, if the patient presents with a likely musculoskeletal etiology of the chest pain, it would be inappropriate to order a computed tomography of the chest evaluating for aortic dissection when a simple chest radiograph may suffice.

Once the learner has performed a history of physical exam, developed a differential diagnosis, determined what laboratory and imaging studies to obtain, the next compartment to be developed should be one of therapeutic intervention. This is perhaps the most difficult of compartments for the new learner since it will require some level of knowledge obtained from long-term memory. It is in this compartment where knowledge and experience most come into play. If the learner comes across a novel presentation of a disease process, intervention by more senior physicians may be required in order for the learner to understand what therapies may be required. After this novel presentation, the learner will then have built this schema into their long-term memory for recall later. Later presentations will become easier to treat.

Finally, for ED settings, the last compartment is that of disposition. What is to be done with the patient? Can they be discharged? Must they be admitted? Therefore, all the compartments generated must be carefully considered to answer this question safely.

This can be created schematically for easy recall: 
Nursing, EMS, Family members

History \& Physical Exam

Differential Diagnosis

Laboratory Investigation

\section{Imaging}

\section{Treatment}

\section{Disposition}

\section{Summary}

Development of a cognitive schema is imperative in this age of medicine due to the pitfalls of multitasking, constant interruptions and the ever-increasing workload placed upon learners. The strategy of compartmentalization may be utilized to aid the learner to facilitate both safe patient care and learning. By utilizing the sensory input gained from the initial history and physical exam, this leads to an initial gestalt that goes back and forth from the working memory to long term memory, thus developing a coherent schema and eventual skillset for the medical learner.

Utilizing this method will mitigate interruptions and distractions, allow experience to be gained, and allow the learner to safely increase their workload all while increasing their experience and knowledge.

\section{References}

Archana, L., Forogh, H., Osman, R. S., Robert, A. G., Zhang, J. J., \& Vimla, L. P. (2007). The Multitasking Clinician: Decision-Making and Cognitive Demand during and after Team Handoffs in Emergency Care. International Journal of Medical Informatics, 76, 801-811. https://doi.org/10.1016/j.ijmedinf.2006.09.019

Blair, R. (2012). 5 Steps of Compartmentalization: The Secret behind Successful Entrepreneurs.

https://www.forbes.com/sites/ryanblair/2012/06/26/5-steps-of-compartmentalization/\# 70dfaead1a62

Bragard, I., Dupuis, G., \& Fleet, R. (2015). Quality of Work Life, Burnout, and Stress in Emergency Department Physicians: A Qualitative Review. European Journal of Emergency Medicine, 22, 227-234. https://doi.org/10.1097/MEJ.0000000000000194

Brown, D. (1995). Stress and Coping Strategies in Community Psychiatric Nurses: A Q-Methodological Study. Journal of Advanced Nursing, 21, 230-237. https://doi.org/10.1111/j.1365-2648.1995.tb02519.x

Chi, M. T. H. (2006). Two Approaches to the Study of Experts' Characteristics. In K. A. Ericsson (Ed.), The Cambridge Handbook of Expertise and Expert Performance (pp. 21-30). Cambridge: Cambridge University Press. https://doi.org/10.1017/CBO9780511816796.002 
Chisholm, C. D., Collison, E. K., Nelson, D. R., \& Cordell, W. H. (2000). Emergency Department Workplace Interruptions: Are Emergency Physicians "Interrupt-Driven" and "Multitasking"? Academic Emergency Medicine, 7, 1239-1243. https://doi.org/10.1111/j.1553-2712.2000.tb00469.x

Counselman, F. L., Babu, K., Edens, M. A., Gorgas, D. L., Hobgood, C., Marco, C. A. et al. (2017). The 2016 Model of the Clinical Practice of Emergency Medicine. Journal of Emergency Medicine, 52, 846-849. https://doi.org/10.1016/j.jemermed.2017.01.040

Croskerry, P. (2000). The Cognitive Imperative Thinking about How We Think. Academic Emergency Medicine, 7, 1223-1231. https://doi.org/10.1111/j.1553-2712.2000.tb00467.x

Croskerry, P., \& Sinclair, D. (2001). Emergency Medicine: A Practice Prone to Error? Canadian Journal of Emergency Medicine, 3, 271-276. https://doi.org/10.1017/S1481803500005765

Duchscher, J. E. B. (2009). Transition Shock: The Initial Stage of Role Adaptation for Newly Graduated Registered Nurses. Journal of Advanced Nursing, 65, 1103-1113. https://doi.org/10.1111/j.1365-2648.2008.04898.x

Ericsson, K. A. (2006). The Cambridge Handbook of Expertise and Expert Performance. Cambridge, NY: Cambridge University Press. https://doi.org/10.1017/CBO9780511816796

Eva, K. W. (2005). What Every Teacher Needs to Know about Clinical Reasoning. Medical Education, 39, 98-106. https://doi.org/10.1111/j.1365-2929.2004.01972.x

Hymel, G., \& Severyn, F. (1999). Typical Shiftwork Interruptions Faced by Supervising EM Faculty in an EM Residency Setting. Abstract Presented at: Society for Academic Emergency Medicine Midwest Regional Meeting, September, Ann Arbor, MI.

Jagsi, R., Weinstein, D. F., Shapiro, J., Kitch, B. T., Dorer, D., \& Weissman, J. S. (2008). The Accreditation Council for Graduate Medical Education's Limits on Residents' Work Hours and Patient Safety. A Study of Resident Experiences and Perceptions before and after Hours Reductions. Archives of Internal Medicine, 168, 493-500. https://doi.org/10.1001/archinternmed.2007.129

Kirmeyer, S. L. (1988). Coping with Competing Demands: Interruption and the Type A Pattern. Journal of Applied Psychology, 73, 621. https://doi.org/10.1037/0021-9010.73.4.621

Kohn, L. T., Corrigan, J. M., \& Donaldson, M. S. (2000). To Err Is Human: Building a Safer Health System: A Report of The Committee on Quality of Health Care in America, Institute of Medicine. Washington DC: National Academy Press.

Michtalik, H. J., Yeh, H. C., Pronovost, P. J., \& Brotman, D. J. (2013). Impact of Attending Physician Workload on Patient Care: A Survey of Hospitalists. JAMA Internal Medicine, 173, 375-377. https://doi.org/10.1001/jamainternmed.2013.1864

Miller, G. A. (1956). The Magical Number Seven, plus or minus Two: Some Limits on Our Capacity for Processing Information. Psychological Review, 63, 81.

https://doi.org/10.1037/h0043158

Rasmussen, J., \& Jensen, A. (1974). Mental Procedures in Real-Life Tasks: A Case Study of Electronic Troubleshooting. Ergonomics, 17, 293-307. https://doi.org/10.1080/00140137408931355

Rhodes, D. R., McFarland, K. F., Finch, W. H., \& Johnson, A. O. (2001). Speaking and Interruptions during Primary Care Office Visits. Family Medicine, 33, 528-532.

Skaugset, L. M., Farrell, S., Carney, M., Wolff, M., Santen, S. A., Perry, M., \& Cico, S. J. (2016). Can You Multitask? Evidence and Limitations of Task Switching and Multi- 
tasking in Emergency Medicine. Annals of Emergency Medicine, 68, 189-195. https://doi.org/10.1016/j.annemergmed.2015.10.003

Tweed, M. J., Bagg, W., Child, S., Wilkinson, T. J., \& Weller, J. M. (2010). How the Trainee Intern Year Can Ease the Transition from Undergraduate Education to Postgraduate Practice. The New Zealand Medical Journal (Online), 123, No. 1318.

Young, J. Q., Van Merrienboer, J., Durning, S., \& Ten Cate, O. (2014). Cognitive Load Theory: Implications for Medical Education: AMEE Guide No. 86. Medical Teacher, 36, 371-384. https://doi.org/10.3109/0142159X.2014.889290 